\author{
ACTA MYCOLOGICA \\ Vol. 47 (2): 175-177 \\ 2012
}

\title{
Introduced Tuber aestivum spreading spontaneously in Israel
}

\author{
VARDA KAGAN-ZUR ${ }^{1 *}$, TIDHAR TURGEMAN ${ }^{2}$, YARON SITRIT $^{2}$, OFER DANAI ${ }^{3}$, \\ YORAM LUZZATI $^{4}$, AMNON BUSTAN ${ }^{2}$, NURIT ROTH-BEJERANO ${ }^{1}$ and SEGULA MASAPHY ${ }^{5}$ \\ ${ }^{1}$ Department of Life Sciences, and ${ }^{2}$ The Jacob Blaustein Institute for Desert Research \\ Ben-Gurion University of the Negev, Beer-Sheva IL-84105 \\ ${ }^{3}$ Edible Mushrooms and ${ }^{5}$ Applied Mycology and Microbiology Departments, \\ MIGAL, P.O. Box 831, Kiryat Shmona IL-11016, and Tel Hai College, Upper Galilee IL-12210 \\ ${ }^{4}$ Kibbutz Bar'am, M.P. Merom Hagalil IL-13860,"corresponding author. zur@bgu.ac.il
}

Kagan-Zur V., Turgeman T., Sitrit Y., Danai O., Luzzati Y., Bustan A., Roth-Bejerano N., Masaphy S.: Introduced Tuber aestivum spreading spontaneously in Israel. Acta Mycol. 47 (2): 175-177, 2012.

A Tuber melanosporum plantation established in 1994/5 on Kibbutz Bar'am (in the Upper Galilee, Israel, Fig. 1) gradually lost its T. melanosporum mycorrhiza. When checked in 1998 , only about $70 \%$ of the trees maintained their original mycorrhiza (Kagan-Zur et al. 2001) and was deteriorating constantly. A number of trees perished. Several different tree species were initially introduced. These included both

Introduced species:

Quercus ilex

Q.pubescens

Corylus avellana

Local species:

Q.boissieri

Q. penduculifora

Q. calliprinus

Q. cerris

Q. libani

Q. ithaburensis

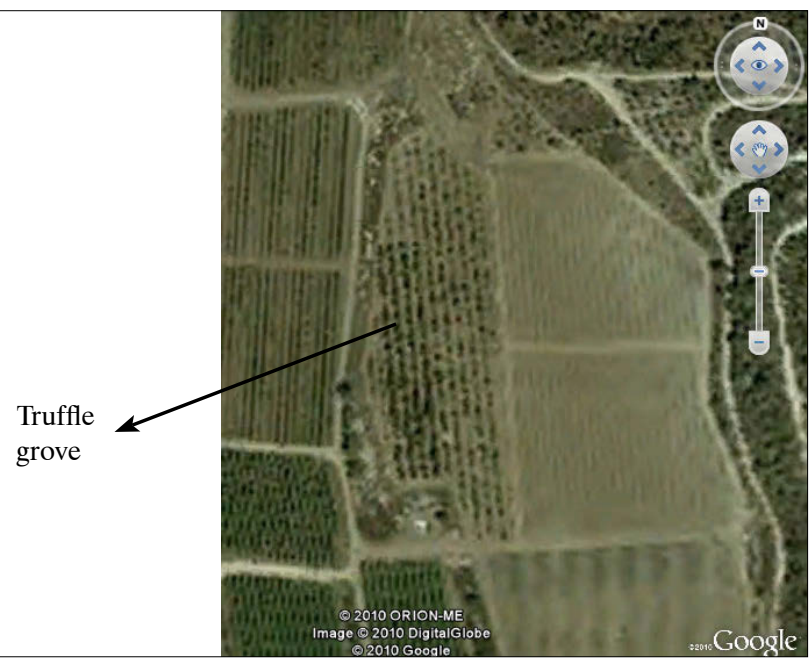

Fig. 1. The plantation (adapted from Google Earth). 

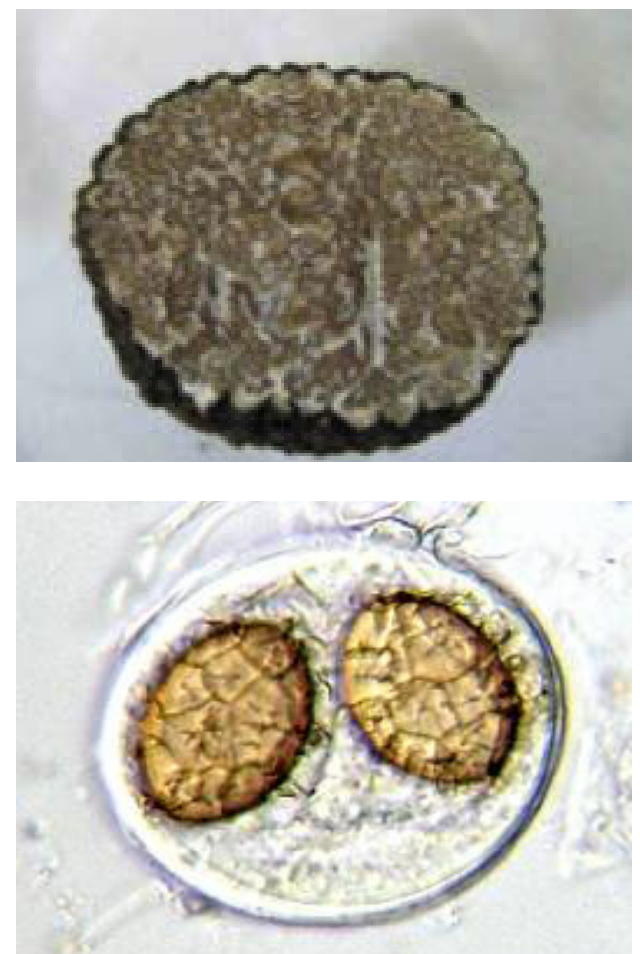

Fig. 2. Tuber aestivum Vittad. On the top: a transverse cut of a fruit body found on 2009 . On the bottom: spores.

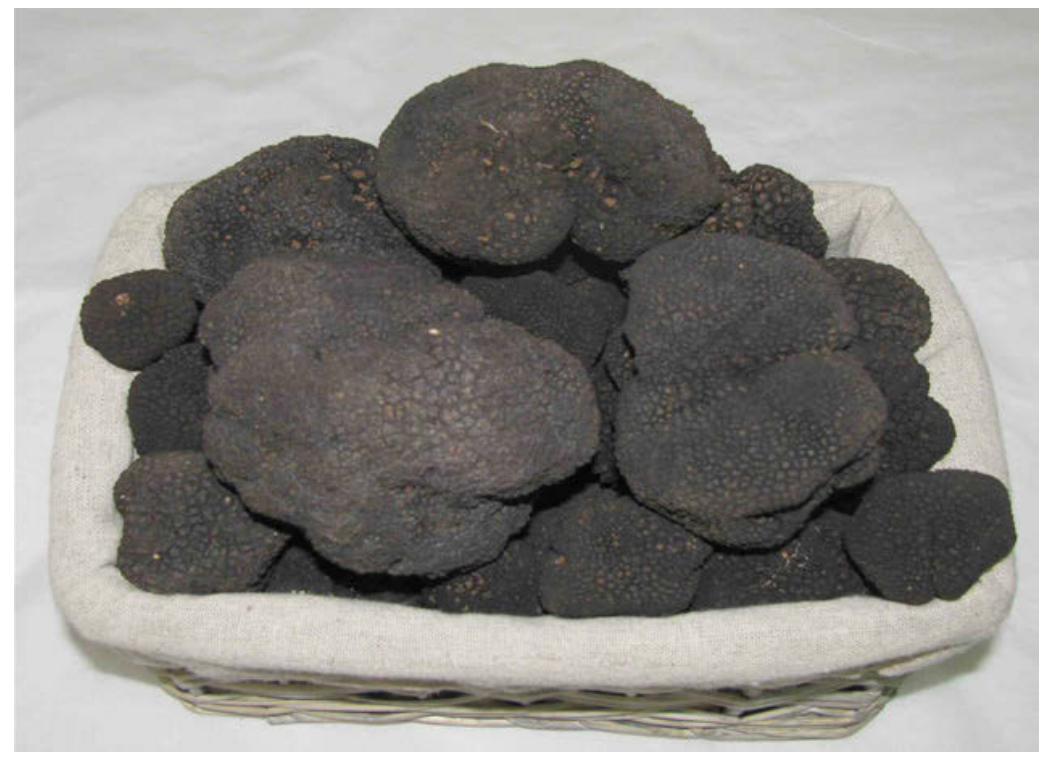

Fig. 3. T. aestivum Vittad. yields. Amount collected in the years: $2010-2.2 \mathrm{~kg}$, $2011-0.0 \mathrm{~kg}, 2012-1.7 \mathrm{~kg}$. 
European and local oak species, as well as hazelnuts. The different tree species were randomly planted within the grove. The plantation setting was necessary to facilitate our identification of the most suitable host species for producing the best yields under Israeli conditions. It was later found that local oak species produced higher yields as compared to introduced oak species, chosen for their reputation of being good T. melanosporum symbionts.

A single T. melanosporum fruit body was collected in 1999 (Kagan-Zur et al. 2000), but none later. In 1999, seedlings were introduced into the plantation to fill the gaps between trees. These included, inadvertently, T. aestivum inoculated plants.

Between 2000 and 2008 no truffles were found. However, in July 2009, two fruit bodies were collected and studied. Their inner colors when cut and spore morphology observed under a light microscope indicated that these fruit bodies had a greater resemblance to T. aestivum than to T. melanosporum (Fig.2). Indeed, molecular analyses proved these to be T. aestivum (Turgeman et al. 2012). Thus, the intentionally introduced T. melanosporum mycorrhiza was replaced by that of another, unintentionally introduced mycorrhizal fungus, T. aestivum. In 2010 an organized manual search was performed (by digging systematically around 125 trees in the plot). A total of $2.2 \mathrm{~kg}$ of fruit bodies for a calculated yield of about $6 \mathrm{~kg} /$ acre was recovered (Fig. 3). This was comparable to yields reported for young commercial orchards elsewhere (Southern Woods Nursery 2011).

No fruit bodies were collected during 2011. However, in 2012 truffles were found both at the original plantation and, accidentally, at a grove within a research farm about $3.5 \mathrm{~km}$ away. The latter seem to be of the same origin as the originally introduced T. aestivum.

In conclusion, the environmental conditions on the Upper Galilee, though unsuitable for T. melanosporum, fit the requirements of the more robust T. aestivum fungus, which thrives on local oak species and even spreads further.

\section{REFERENCES}

Kagan Zur V., Freeman S., Luzzati Y., Roth-Bejerano N., Shabi E. 2000. Emergence of the first black Périgord truffle (Tuber melanosporum) in Israel. Mycol Veget. Mediter. 15: 187-192.

Kagan-Zur V., Freeman S., Luzzati Y., Roth-Bejerano N., Shabi E. 2001. Survival of introduced Tuber melanosporum mycorrhizas at two sites in Israel as measured by its occurrence on mycorrhizas. Plant \& Soil 229: 159-166.

Southern Woods Nursery: Truffles. 2011. http://www.southernwoods.co.nz/documents/Truffles.pdf

Turgeman T., Sitrit Y., Danai O., Luzzati Y., Bustan A., Roth-Bejerano N., Kagan-Zur V., Masaphy S. 2012. Introduced Tuber aestivum replacing introduced Tuber melanosporum: a case study. Agroforestry Systems 84: 337-343. 